\title{
Otimização Dinâmica de uma Planta de Gaseifica- ção para Geração de Combustível
}

Silva, F. R.

Escola Politécnica de Pernambuco

Universidade de Pernambuco

50.720-001 - Recife, Brasil

engmec_rodrigues@hotmail.com
Silva, J. D.

Escola Politécnica de Pernambuco

Universidade de Pernambuco

50.720-001 - Recife, Brasil

Resumo Oalcatrão formado durante o processo de gaseificação de biomassa é um composto indesejável que prejudica o rendimento do processo e danifica equipamentos. O foco deste trabalho é o estudo da otimização da reação de eliminação do alcatrão através do processo catalítico utilizando-se um catalisador à base de níquel. Através do modelo matemático proposto, os componentes do processo de craqueamento do alcatrão serão analisados, quantificando-se produção e consumo e o comportamento das temperaturas nas fases gasosa e sólida para um reator de leito fixo.

Abs- $\quad$ The tar formed during the gasification of biomass is an undesirable compound that interferes with tract the performance of the process and damage equipment. The focus of this paper is to study the optimization of tar elimination reaction through a catalytic process using a catalyst based on nickel. Through the mathematical model, the components of the tar cracking process will be analyzed, quantifying the production and consumption and the behavior of temperatures in gaseous and solid phases for a fixed bed reactor. 


\section{Introdução}

Em tempos de alta demanda energética e desenvolvimento sustentável, diversas tecnologias alternativas de geração de energia são desenvolvidas. A gaseificação de biomassa tem se mostrado um dos principais e mais eficientes processos renováveis de geração de energia.

Durante a gaseificação, a biomassa, através de reações com ar, oxigênio ou vapor de água é convertida em uma mistura de gases denominada gás de síntese, já que este servirá de matéria-prima na fabricação de combustíveis. Além dos gases há também a formação de uma pequena quantidade de hidrocarbonetos voláteis juntamente com o alcatrão [1]. O alcatrão formado durante o processo de gaseificação pode ser definido como todo contaminante orgânico com peso molecular maior do que o do benzeno [2]. Porém, esta é apenas uma das diversas definições dadas a este composto, já que para defini-lo com base em sua composição química fatores como variação da composição do produto gasoso final desejado para uma aplicação particular e de como o alcatrão é coletado e analisado devem ser levados em consideração. O alcatrão é um composto indesejável, pois pode condensar ou polimerizar-se em estruturas mais complexas nas tubulações, filtros e trocadores de calor, danificando equipamentos, diminuindo a eficiência total e aumentando o custo do processo [3]. Um dos principais desafios para a otimização da gaseificação de biomassa é como eliminar o alcatrão produzido durante o processo. A aplicação de catalisadores no leito do gaseificador a altas temperaturas serve para oxidar a molécula de alcatrão, gerando moléculas menores e mais simples e ainda $\mathrm{H}_{2}$ e CO, principais componentes do produto gasoso final. Os tipos de catalisadores mais utilizados e pesquisados para este fim são a dolomita calcinada, olivina e catalisadores a base de $\mathrm{Ni}$, sendo o mais eficiente o catalisador a base de níquel. O objetivo deste trabalho é caracterizar o processo de otimização da gaseificação de biomassa através do craqueamento do alcatrão utilizando catalisador a base de níquel. Para isso será considerada a seguinte reação:

$$
2 \mathrm{C}_{7} \mathrm{H}_{8}+7 \mathrm{O}_{2} \stackrel{\mathrm{N}^{2} / \mathrm{Al}_{2} \mathrm{O}_{3}}{-} 8 \mathrm{H}_{2}+14 \mathrm{CO}
$$

O tolueno foi usado como composto modelo de alcatrão, porque representa uma estrutura aromática estável resultante em alcatrões formados em processo a altas temperaturas [4]. Através do modelo matemático desenvolvido serão analisados os perfis de temperatura e de transferência de massa para todos os componentes da reação tanto em função do tempo como em função do comprimento do reator.

\section{Modelagem Matemática}

Neste trabalho o modelo matemático para a reforma catalítica do alcatrão foi desenvolvido através das equações de conservação de energia e massa, assim desenvolveu-se um conjunto de equações que caracteriza a transferência de energia e massa para o fenômeno da reforma do alcatrão cuja solução descreve os perfis de temperatura e fração molar para todos os componentes da reação estudada. As equações gerais que descrevem o fenômeno da reforma catalítica do alcatrão são as seguintes:

- Balanço de energia para a fase vapor:

$$
\begin{aligned}
& \left(1-\varepsilon_{\mathrm{s}}\right) \rho_{\mathrm{v}} \mathrm{C}_{\mathrm{p}, \mathrm{v}} \frac{\partial \mathrm{T}_{\mathrm{v}}}{\partial \mathrm{t}}+\rho_{\mathrm{v}} \mathrm{C}_{\mathrm{p}, \mathrm{v}} \mathrm{V}_{\mathrm{v}} \frac{\partial \mathrm{T}_{\mathrm{v}}}{\partial \mathrm{z}}= \\
& \left(1-\varepsilon_{\mathrm{s}}\right) \lambda_{\mathrm{ax}, \mathrm{v}} \frac{\partial^{2} \mathrm{~T}_{\mathrm{v}}}{\partial \mathrm{z}^{2}}-\left(\alpha_{\mathrm{vs}} \eta_{\mathrm{CE}} \varepsilon_{\mathrm{s}} \mathrm{a}_{\mathrm{s}}\right)\left[\mathrm{T}_{\mathrm{v}}-\mathrm{T}_{\mathrm{s}}\right] \\
& +\alpha_{\mathrm{vw}}\left(1-\eta_{\mathrm{EC}}\right) \mathrm{a}_{\mathrm{w}}\left[\mathrm{T}_{\mathrm{v}}-\mathrm{T}_{\mathrm{w}}\right]
\end{aligned}
$$

- Condições iniciais de contorno:

$$
\begin{aligned}
& \left.\mathrm{T}_{\mathrm{v}}\right|_{\mathrm{t}=0}=\mathrm{T}_{\mathrm{v}, \mathrm{e}} \\
& \left.\left(1-\varepsilon_{\mathrm{s}}\right) \lambda_{\mathrm{ax}, \mathrm{v}} \frac{\partial \mathrm{T}_{\mathrm{v}}}{\partial \mathrm{z}}\right|_{\mathrm{z}-0^{+}}= \\
& \rho_{\mathrm{v}} \mathrm{C}_{\mathrm{p}, \mathrm{v}} \mathrm{V}_{\mathrm{v}}\left[\left.\mathrm{T}_{\mathrm{v}}\right|_{\mathrm{z}-0^{+}}-\mathrm{T}_{\mathrm{v}, \mathrm{e}}\right] \\
& \left.\frac{\partial \mathrm{T}_{\mathrm{v}}}{\partial \mathrm{z}}\right|_{\mathrm{z}=\mathrm{L}}=0
\end{aligned}
$$

- Balanço de energia para fase sólida:

$$
\begin{aligned}
& \varepsilon_{s} \rho_{s} C_{p, s} \frac{\partial T_{s}}{\partial t}=\varepsilon_{s} \lambda_{a x, s} \frac{\partial^{2} T_{s}}{\partial z^{2}} \\
& +\left(\alpha_{v s} M_{C E} \varepsilon_{s} a_{s}\right)\left[T_{v}-T_{s}\right]+\varepsilon_{s} r_{x}^{\prime}\left(-\Delta H_{r}\right)
\end{aligned}
$$

- Condições iniciais e de contorno: 


$$
\begin{aligned}
& \left.\mathrm{T}_{\mathrm{s}}\right|_{\mathrm{t}=0}=\mathrm{T}_{\mathrm{s}, \mathrm{e}} \\
& \left.\varepsilon_{\mathrm{s}} \lambda_{\mathrm{ax}, \mathrm{s}} \frac{\partial \mathrm{T}_{\mathrm{s}}}{\partial \mathrm{z}}\right|_{\mathrm{z}=0^{+}}=\mathrm{q}_{\mathrm{c}} \\
& \left.\frac{\partial \mathrm{T}_{\mathrm{s}}}{\partial \mathrm{z}}\right|_{\mathrm{z}=\mathrm{L}}=0
\end{aligned}
$$

- Balanço de massa para o tolueno:

$h_{t} \frac{\partial C_{t}}{\partial t}+\frac{4 Q_{g}}{\pi d^{2}} \frac{\partial C_{t}}{\partial z}=D_{t} \frac{\partial^{2} C_{t}}{\partial z^{2}}$
$+\varepsilon_{s} M_{t} R_{t}$

- Condições iniciais e de contorno para o tolueno:

$$
\begin{aligned}
& \left.C_{t}\right|_{t-0}=C_{t, 0} \\
& \left.D_{t} \frac{\partial C_{t}}{\partial z}\right|_{z=0^{+}}=\frac{4 Q_{g}}{\pi d^{2}}\left[\left.C_{t}\right|_{z=0^{+}}-C_{t, 0}-\right. \\
& \left.\frac{\partial C_{t}}{\partial z}\right|_{z=1}=0
\end{aligned}
$$

- Balanço de massa para o $\mathrm{O}_{2}$ :

$$
\begin{aligned}
& \mathrm{h}_{\mathrm{t}} \frac{\partial \mathrm{C}_{\mathrm{O}_{2}}}{\partial \mathrm{t}}+\frac{4 \mathrm{Q}_{\mathrm{g}}}{\pi \mathrm{d}^{2}} \frac{\partial \mathrm{C}_{\mathrm{O}_{2}}}{\partial \mathrm{z}}=\mathrm{D}_{\mathrm{O}_{2}} \frac{\partial^{2} \mathrm{C}_{\mathrm{O}_{2}}}{\partial \mathrm{z}^{2}} \\
& +\varepsilon_{\mathrm{s}} \mathrm{M}_{\mathrm{O}_{2}} \mathrm{R}_{\mathrm{O}_{2}}
\end{aligned}
$$

- Condições iniciais e de contorno para o $\mathrm{O}_{2}$

$$
\begin{aligned}
& \left.\mathrm{C}_{\mathrm{O}_{2}}\right|_{\mathrm{t}=0}=\mathrm{C}_{\mathrm{O}_{2}, 0} \\
& \left.\mathrm{D}_{\mathrm{O}_{2}} \frac{\partial \mathrm{C}_{\mathrm{O}_{2}}}{\partial \mathrm{z}}\right|_{\mathrm{z}=0^{+}}=\left.\frac{4 \mathrm{Q}_{\mathrm{g}}}{\pi \mathrm{d}^{2}} \mathrm{C}_{\mathrm{O}_{2}}\right|_{\mathrm{z}=0^{+}}-\mathrm{C}_{\mathrm{O}_{2}, 0}-
\end{aligned}
$$

$$
\left.\frac{\partial \mathrm{C}_{\mathrm{O}_{2}}}{\partial \mathrm{z}}\right|_{\mathrm{z}-1}=0
$$

- Balanço de massa para o CO:

$\mathrm{h}_{\mathrm{t}} \frac{\partial \mathrm{C}_{\mathrm{CO}}}{\partial \mathrm{t}}+\frac{4 \mathrm{Q}_{\mathrm{g}}}{\pi \mathrm{d}^{2}} \frac{\partial \mathrm{C}_{\mathrm{CO}}}{\partial \mathrm{z}}=\mathrm{D}_{\mathrm{CO}} \frac{\partial^{2} \mathrm{C}_{\mathrm{CO}}}{\partial \mathrm{z}^{2}}$

$$
+\varepsilon_{\mathrm{s}} \mathrm{M}_{\mathrm{CO}} \mathrm{R}_{\mathrm{CO}}
$$

- Condições iniciais e de contorno para o CO:

$$
\left.\mathrm{C}_{\mathrm{CO}}\right|_{\mathrm{t}=0}=\mathrm{C}_{\mathrm{CO}, 0}
$$

$\left.\mathrm{D}_{\mathrm{CO}} \frac{\partial \mathrm{C}_{\mathrm{CO}}}{\partial \mathrm{z}}\right|_{\mathrm{z}=0^{+}}=\frac{4 \mathrm{Q}_{\mathrm{g}}}{\pi \mathrm{d}^{2}}\left[\left.\mathrm{C}_{\mathrm{CO}}\right|_{\mathrm{z}=0^{+}}-\mathrm{C}_{\mathrm{CO}, 0}-\right.$

$\left.\frac{\partial \mathrm{C}_{\mathrm{CO}}}{\partial \mathrm{z}}\right|_{\mathrm{z}-1}=0$

- Balanço de massa para o $\mathrm{H}_{2}$ :

$$
\begin{aligned}
& \mathrm{h}_{\mathrm{t}} \frac{\partial \mathrm{C}_{\mathrm{H}_{2}}}{\partial \mathrm{t}}+\frac{4 \mathrm{Q}_{\mathrm{g}}}{\pi \mathrm{d}^{2}} \frac{\partial \mathrm{C}_{\mathrm{H}_{2}}}{\partial \mathrm{z}}=\mathrm{D}_{\mathrm{H}_{2}} \frac{\partial^{2} \mathrm{C}_{\mathrm{H}_{2}}}{\partial \mathrm{z}^{2}}+ \\
& \varepsilon_{\mathrm{s}} \mathrm{M}_{\mathrm{H}_{2}} \mathrm{R}_{\mathrm{H}_{2}}
\end{aligned}
$$

- Condições iniciais e de contorno para o $\mathrm{H}_{2}$ :

$$
\begin{gathered}
\left.\mathrm{C}_{\mathrm{H}_{2}}\right|_{\mathrm{t}=0}=\mathrm{C}_{\mathrm{H}_{2}, 0} \\
\left.\mathrm{D}_{\mathrm{H}_{2}} \frac{\partial \mathrm{C}_{\mathrm{H}_{2}}}{\partial \mathrm{z}}\right|_{\mathrm{z}=0^{+}}=\frac{4 \mathrm{Q}_{\mathrm{g}}}{\pi \mathrm{d}^{2}}\left[\left.\mathrm{C}_{\mathrm{H}_{2}}\right|_{\mathrm{z}-0^{+}}-\mathrm{C}_{\mathrm{H}_{2}, 0}-\right. \\
\left.\frac{\partial \mathrm{C}_{\mathrm{H}_{2}}}{\partial \mathrm{z}}\right|_{\mathrm{z}=1}=0
\end{gathered}
$$

As equações de (1) a (24) podem ser simplificadas segundo as seguintes hipóteses simplificadoras: (i) modelo 
unidimensional, (ii) sistema não-isotérmico, (iii) as temperaturas da fase gasosa e sólida são modeladas através de modelos de dispersão térmica e (iv) os balanços de massa para a fase gasosa e sólida são modelados levando-se em consideração os efeitos de dispersão axial. Baseado nessas hipóteses, o modelo matemático simplificado para a reforma catalítica do alcatrão é formado pelas equações simplificadas de transporte energia e massa que descrevem o comportamento das temperaturas e frações molares. Assim, as equações simplificadas que formam o modelo são expressas por:

- Balanço de energia para a fase vapor:

$$
\begin{aligned}
& \frac{\mathrm{dT}_{\mathrm{v}}}{\mathrm{dt}}=\alpha_{13, \mathrm{v}}\left(\mathrm{T}_{\mathrm{v}}\right)_{\mathrm{j}}^{(\mathrm{k})}+\alpha_{3, \mathrm{v}}\left(\mathrm{T}_{\mathrm{s}}\right)_{\mathrm{j}}^{(\mathrm{k})}- \\
& \alpha_{4, \mathrm{v}}\left(\mathrm{T}_{\mathrm{w}}\right)_{\mathrm{j}}^{(\mathrm{k})}+\alpha_{14, \mathrm{v}}
\end{aligned}
$$

- Balanço de energia para a fase sólida:

$$
\frac{\mathrm{dT}_{\mathrm{s}}}{\mathrm{dt}}=\alpha_{21, \mathrm{~s}}\left(\mathrm{~T}_{\mathrm{s}}\right)_{\mathrm{j}}^{(\mathrm{k})}+\alpha_{16, \mathrm{~s}}\left(\mathrm{~T}_{\mathrm{v}}\right)_{\mathrm{j}}^{(\mathrm{k})}+\alpha_{22, \mathrm{~s}}
$$

- Balanço de a massa do tolueno:

$$
\frac{\mathrm{dC}_{\mathrm{t}}}{\mathrm{dt}}=\alpha_{12, \mathrm{t}}\left(\mathrm{C}_{\mathrm{t}}\right)_{\mathrm{j}}^{(\mathrm{k})}+\alpha_{13, t}
$$

- $\quad$ Balanço de massa para o $\mathrm{O}_{2}$

$$
\frac{\mathrm{dC}_{\mathrm{O}_{2}}}{\mathrm{dt}}=\alpha_{12, \mathrm{O}_{2}}\left(\mathrm{C}_{\mathrm{O}_{2}}\right)_{\mathrm{j}}^{(\mathrm{k})}+\alpha_{13, \mathrm{O}_{2}}
$$

- Balanço de massa para o CO:

$$
\frac{\mathrm{dC}_{\mathrm{CO}}}{\mathrm{dt}}=\alpha_{12, \mathrm{CO}}\left(\mathrm{C}_{\mathrm{CO}}\right)_{\mathrm{j}}^{(\mathrm{k})}+\alpha_{13, \mathrm{CO}}
$$

- Balanço de massa para $\mathrm{H}_{2}$ :

$$
\frac{\mathrm{dC}_{\mathrm{H}_{2}}}{\mathrm{dt}}=\alpha_{12, \mathrm{H}_{2}}\left(\mathrm{C}_{\mathrm{H}_{2}}\right)_{\mathrm{j}}^{(\mathrm{k})}+\alpha_{13, \mathrm{H}_{2}}
$$

A simplificação das equações gerou um conjunto de parâmetros $\alpha$ que estão apresentados nas tabelas 1 a 5 do apêndice $\mathrm{A}$.

\section{Resultados e discussões}

Nesta seção, os resultados da simulação do modelo serão apresentados. A resolução do modelo mostra o perfil do comportamento da temperatura nas fases gasosa e sólida e fração molar para todos os componentes envolvidos na reação de reforma catalítica do alcatrão. Estas equações foram resolvidas usando o Método de Euler, na sequência, foi desenvolvido um programa computacional na linguagem FORTRAN para fornecer os resultados das variáveis $\mathrm{Tfg}$, Tfs, $\mathrm{C} 7 \mathrm{H} 8, \mathrm{O} 2, \mathrm{CO}$ e $\mathrm{H} 2$. O programa recebeu parâmetros de experimentos independentes mostrados na Tabela 1.

Tabela 1. Parâmetros para simulação

\begin{tabular}{|c|c|}
\hline$\varepsilon_{\mathrm{s}}=0,72$ & $\mathrm{a}_{\mathrm{w}}=0,57 \mathrm{~m}^{2}$ \\
\hline$\rho_{\mathrm{v}}=0,956 \mathrm{~kg} / \mathrm{m}^{3}$ & $\mathrm{q}_{\mathrm{c}}=208 \mathrm{~kJ} / \mathrm{s}$ \\
\hline $\mathrm{C}_{\mathrm{p}, \mathrm{v}}=32 \mathrm{~J} / \mathrm{mol} \mathrm{K}$ & $\mathrm{h}_{\mathrm{t}}=0,46$ \\
\hline $\mathrm{V}_{\mathrm{v}}=0,021 \mathrm{~m} / \mathrm{s}$ & $\mathrm{Q}_{\mathrm{g}}=2,32 \times 10^{-3} \mathrm{~m}^{3} / \mathrm{s}$ \\
\hline$\lambda_{\mathrm{ax}, \mathrm{v}}=2,09 \mathrm{~J} / \mathrm{m}^{2} \mathrm{~s} \mathrm{~K}$ & $\mathrm{~d}=1 \mathrm{~m}$ \\
\hline$\alpha_{\mathrm{vs}}=1,81 \mathrm{~W} / \mathrm{m}^{2} \mathrm{~K}$ & $\mathrm{D}_{\mathrm{t}}=1,25 \times 10^{-5} \mathrm{~m}^{2} / \mathrm{s}$ \\
\hline$\eta_{\mathrm{ce}}=0,35$ & $\mathrm{D}_{\mathrm{O} 2}=3,24 \times 10^{-5} \mathrm{~m}^{2} / \mathrm{s}$ \\
\hline $\mathrm{a}_{\mathrm{s}}=156 \mathrm{~m}^{-1}$ & $\mathrm{D}_{\mathrm{co}}=4,61 \times 10^{-6} \mathrm{~m}^{2} / \mathrm{s}$ \\
\hline$\rho_{\mathrm{s}}=1250 \mathrm{~kg} / \mathrm{m}^{3}$ & $\mathrm{D}_{\mathrm{H} 2}=6,52 \times 10^{-6} \mathrm{~m}^{2} / \mathrm{s}$ \\
\hline $\mathrm{C}_{\mathrm{p}, \mathrm{s}}=18 \mathrm{~J} / \mathrm{mol} \mathrm{K}$ & $\eta_{\mathrm{t}}=0,95$ \\
\hline$\lambda_{\mathrm{ax}, \mathrm{s}}=1,79 \mathrm{~J} / \mathrm{m}^{2} \mathrm{~s} \mathrm{~K}$ & $\eta_{\mathrm{O} 2}=0,65$ \\
\hline$\Delta \mathrm{H}_{\mathrm{r}}=-283 \mathrm{~kJ} / \mathrm{mol}$ & $\eta_{\mathrm{CO}}=0,79$ \\
\hline$\alpha_{\mathrm{vw}}=0,12$ & $\eta_{\mathrm{H} 2}=0,86$ \\
\hline \multicolumn{2}{|}{}
\end{tabular}

Os resultados serão mostrados em sua forma gráfica. A figura 1 caracteriza os perfis de temperatura para as fases gasosa e sólida, sendo $\mathrm{O}$ aumento da temperatura tanto da fase sólida como da fase gasosa devido à agitação molecular dentro do gaseificador. 


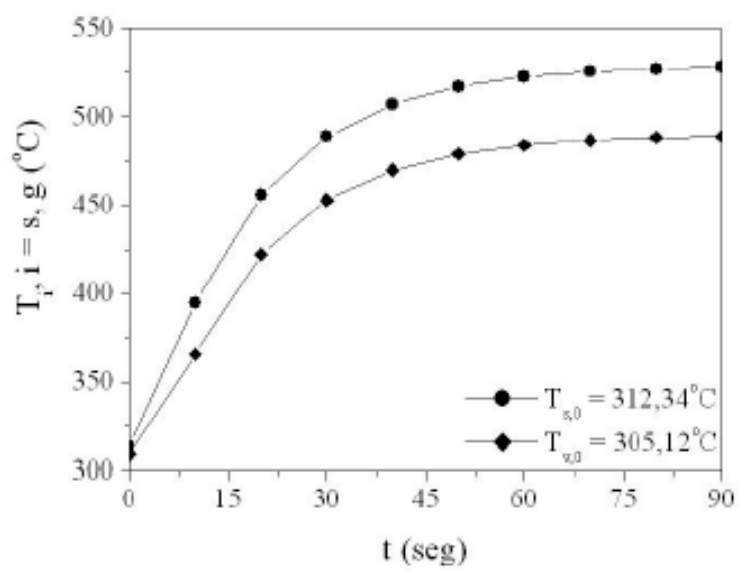

Fig 1. Perfis de temperatura para a fase vapor $\left(T_{v}\right)$ e para a fase sólida $\left(T_{s}\right)$

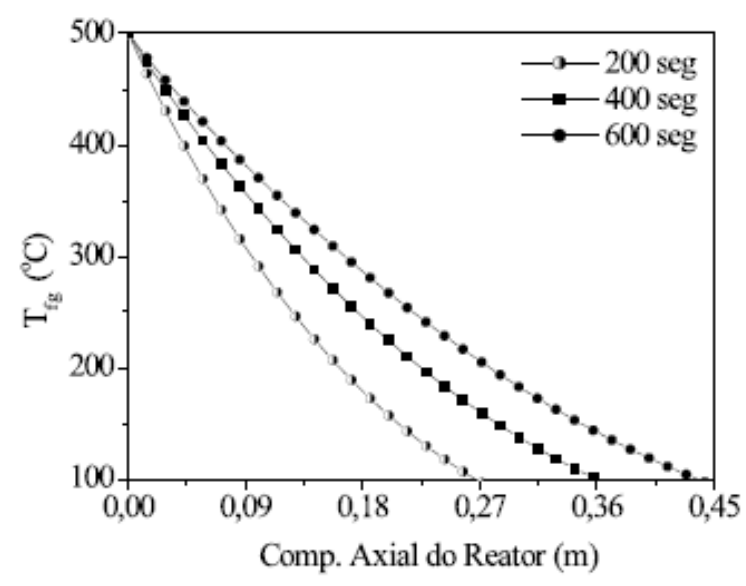

Fig 2. Perfil de temperatura para a fase vapor $\left(\mathrm{T}_{\mathrm{fg}}\right.$ ) em função do comprimento axial do reator.

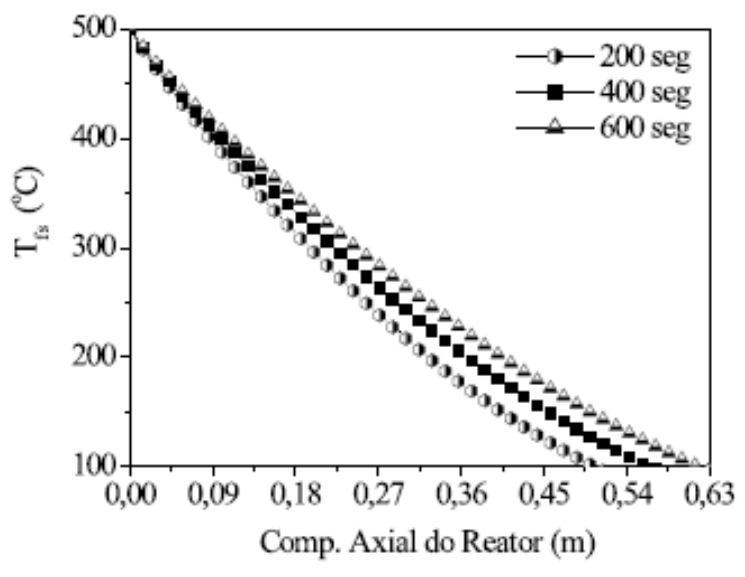

Fig 3. Perfis de temperatura para a fase sólida $\left(\mathrm{T}_{\mathrm{fs}}\right)$ em função do comprimento axial do reator.

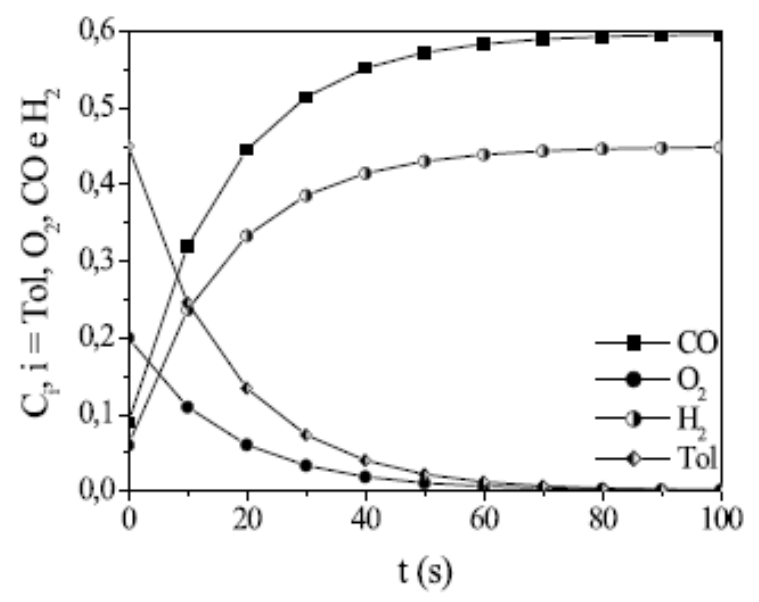

Fig 4. Perfis de concentrações das espécies químicas $\mathrm{C}_{7} \mathrm{H}_{8}, \mathrm{O}_{2}, \mathrm{CO}$ e $\mathrm{H}_{2}$, na saída do reator catalítico trifásico de leito fixo. 


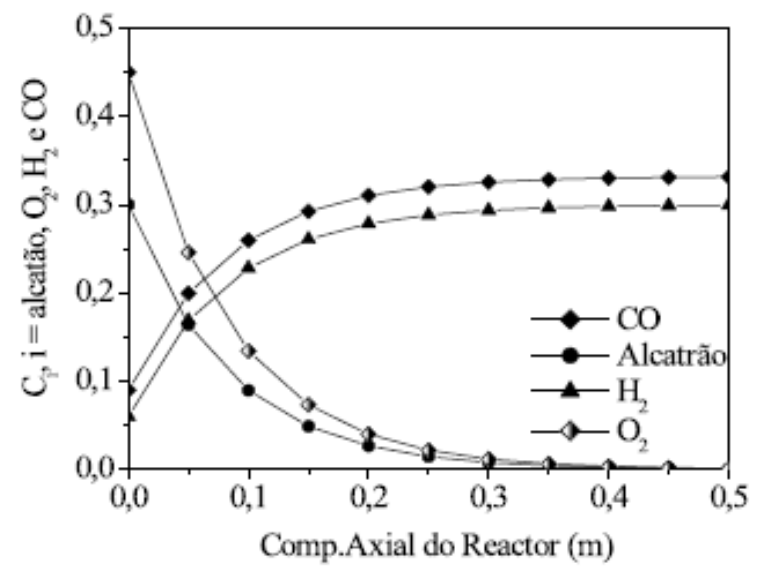

Fig 5. Comportamento dos componentes gasoso em função do comprimento axial do reator de leito fixo

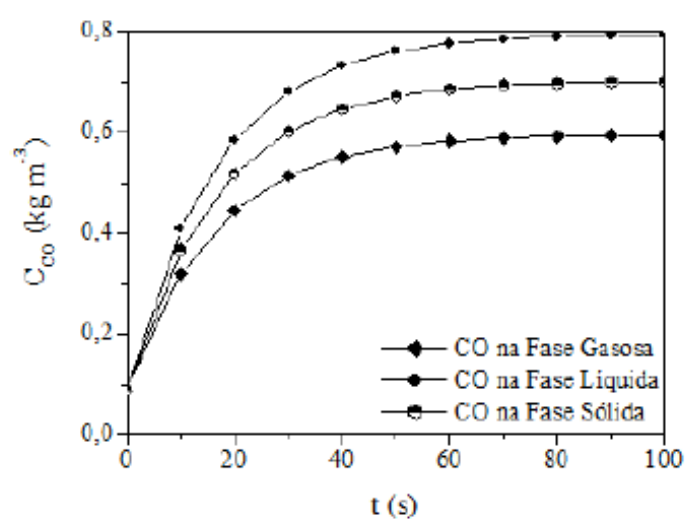

Fig 6. Perfis da concentração do componente $\mathrm{CO}$ nas fases gasosa, líquida e sólida do reator trifásico catalítico de leito fixo

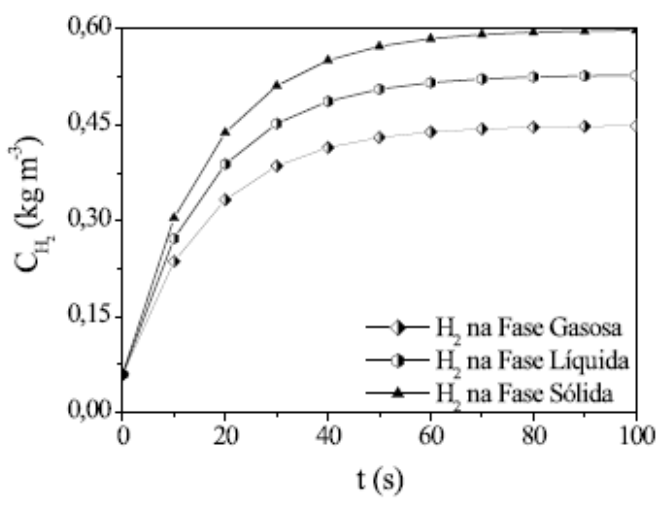

Fig 7. Perfis da concentração do componente $\mathrm{H}_{2}$ nas fases gasosa, líquida e sólida

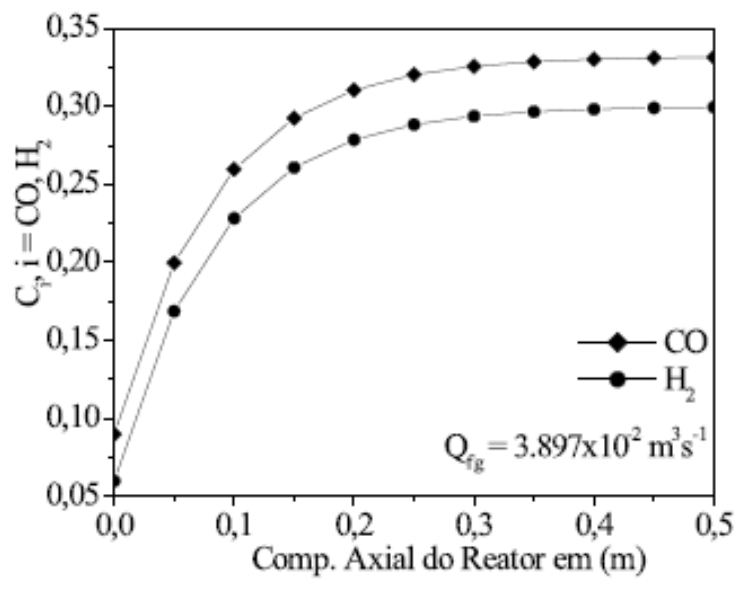

Fig 8. Produção dos componentes $\mathrm{H}_{2}$ e $\mathrm{CO}$ em função do comprimento axial do reator de leito fixo

\section{Conclusões}

O estudo mostrado no presente trabalho permitiu analisar os comportamentos da temperatura do fluido gasoso, da temperatura na fase sólida e da fração molar das espécies químicas $\mathrm{C} 7 \mathrm{H} 8, \mathrm{O} 2, \mathrm{CO}$ e $\mathrm{H} 2$ em função do tempo e do comprimento do reator. A partir dos resultados mostrados, pode-se apresentar as seguintes conclusões:

- A temperatura da fase vapor aumenta estabilizando-se em $\pm 487,5^{\circ} \mathrm{C}$;

- A temperatura da fase sólida aumenta estabilizase em $\pm 525^{\circ} \mathrm{C}$;

- Após 600 segundos de reação a temperatura da fase gasosa chega a $100^{\circ} \mathrm{C}$ a $0,45 \mathrm{~m}$ do comprimento do reator;

- Após 600 segundos de reação, a temperatura da fase sólida chega a $100^{\circ} \mathrm{C}$ a $0,63 \mathrm{~m}$ do comprimento do reator;

- A concetração de $\mathrm{CO}$ cresce conforme o tolueno e o $\mathrm{O} 2$ são consumidos e estabilizasse $\mathrm{em} \pm 0,6$ $\mathrm{Kg}$;

- A concentração de $\mathrm{H} 2$ cresce conforme o tolueno e o O2 são consumidos e estabilizasse em $\pm 0,45$ $\mathrm{Kg}$; 
- A concentração de $\mathrm{CO}$ aumenta enquanto o alcatrão e o $\mathrm{O} 2$ são consumidos e estabiliza-se em \pm $0,325 \mathrm{Kg} / \mathrm{m}^{3}$ a $0,375 \mathrm{~m}$ do comprimento do reator;

- A concentração de H2 aumenta enquanto o alcatrão e o $\mathrm{O} 2$ são consumidos e estabiliza-se a \pm $0,375 \mathrm{~m}$ do comprimento do reator;

- As concentrações de $\mathrm{CO}$ e $\mathrm{H} 2$ estabilizamse a \pm $0,45 \mathrm{~m}$ do comprimento do reator.

\section{Notações}

$\mathrm{C}_{\mathrm{p}, \mathrm{v}}$ Capacidade calorífica da fase vapor de tolueno, $\mathrm{J} / \mathrm{mol} \mathrm{K}$

$\mathrm{C}_{\mathrm{p}, 8} \quad$ Capacidade calorífica da fase sólida, $\mathrm{J} / \mathrm{mol} \mathrm{K}$

$\mathrm{V}_{\mathrm{v}} \quad$ Velocidade da fase vapor, $\mathrm{m} / \mathrm{s}$

$\mathrm{a}_{\mathrm{s}} \quad$ Área específica por volume, $\mathrm{m}^{2} / \mathrm{m}^{3}$

$\mathrm{T}_{\mathrm{v}} \quad$ Temperatura da fase vapor, $\mathrm{K}$

$\mathrm{T}_{\mathrm{s}} \quad$ Temperatura da fase sólida, $\mathrm{K}$

$\mathrm{T}_{\mathrm{w}} \quad$ Temperatura da parede, $\mathrm{K}$

$\mathrm{T}_{\mathrm{v}, l} \quad$ Temperatura do vapor na saída do reator, $\mathrm{K}$

$\mathrm{a}_{\mathrm{w}} \quad$ Área da parede do reator, $\mathrm{m}^{2}$

$\mathrm{r}_{\mathrm{x}}$ $\quad$ Taxa de reação, $\mathrm{mol} / \mathrm{s} \mathrm{m}^{3}$

$-\Delta \mathrm{H}_{\mathrm{r}} \quad$ Entalpia da reação,k $\mathrm{J} / \mathrm{mol}$

$\mathrm{q}_{\mathrm{c}} \quad$ Fluxo de calor na entrada do reator, $\mathrm{kJ} / \mathrm{s}$

$h_{t} \quad$ Coeficiente de retenção do tolueno, (-)

$h_{o 2}$ Coeficiente de retenção do oxigênio, (-)

$h_{c o} \quad$ Coeficiente de retenção do óxido de carbono, (-)

$h_{h 2} \quad$ Coeficiente de retenção do hidrogênio, (-)

$Q_{g} \quad$ Vazão do gás, $\mathrm{m}^{3} / \mathrm{s}$

d Diâmetro do reator, $m$

$\mathrm{D}_{\mathrm{t}} \quad$ Coeficiente molecular de difusão do tolueno, $\mathrm{m}^{2} / \mathrm{s}$

$\mathrm{D}_{\mathrm{o} 2}$ Coeficiente molecular de difusão do oxigênio, $\mathrm{m}^{2} / \mathrm{s}$
$\mathrm{D}_{\mathrm{co}} \quad$ Coeficiente molecular de difusão do óxido de carbono, $\mathrm{m}^{2} / \mathrm{s}$

$\mathrm{D}_{\mathrm{h} 2}$ Coeficiente molecular de difusão do hidrogênio, $\mathrm{m}^{2} / \mathrm{s}$

$\mathrm{R}_{\mathrm{t}} \quad$ Taxa de reação para o tolueno, $1 / \mathrm{s}$

$\mathrm{R}_{02}$ Taxa de reação para o oxigênio, $1 / \mathrm{s}$

$\mathrm{R}_{\mathrm{co}} \quad$ Taxa de reação para o monóxido de carbono, $1 / \mathrm{s}$

$\mathrm{R}_{\mathrm{H} 2}$ Taxa de reação para o hidrogênio, $1 / \mathrm{s}$

\section{Letras Gregas}

$\varepsilon_{\mathrm{s}} \quad$ Porosidade da fase sólida, (-)

$\rho_{\mathrm{v}} \quad$ Densidade da fase vapor de tolueno, $\mathrm{Kg} / \mathrm{m}^{3}$

$\rho_{\mathrm{s}} \quad$ Densidade da fase sólida, $\mathrm{Kg} / \mathrm{m}^{3}$

$\lambda_{\mathrm{ax}, \mathrm{v}}$ Dispersão térmica do vapor de tolueno, $\mathrm{J} / \mathrm{m} 2 \mathrm{~s} \mathrm{~K}$

$\lambda_{\mathrm{ax}, \mathrm{s}} \quad$ Dispersão térmica da fase sólida, $\mathrm{J} / \mathrm{m} 2 \mathrm{sK}$

$\alpha_{\mathrm{v}, \mathrm{s}} \quad$ Coeficiente de transferência vapor de tolueno-sólido, $\mathrm{W} / \mathrm{m}^{2} \mathrm{~K}$

$\eta_{\mathrm{CE}} \quad$ Eficiência de molhabilidade externa, (-)

$\eta_{t} \quad$ Fator de efetividade do tolueno, (-)

$\eta_{02} \quad$ Fator de efetividade do oxigênio, (-)

псO Fator de efetividade do monóxido de carbono, (-)

$\eta_{\text {H2 }} \quad$ Fator de efetividade do hidrogênio, (-)

$\alpha_{\mathrm{v}, \mathrm{w}} \quad$ Coeficiente de transferência na parede, (-)

\section{Agradecimentos}

Os autores deste artigo gostariam de agradecer ao CNPQ (Conselho Nacional de Desenvolvimento Científico e Tecnológico) pelo suporte financeiro dado. (Processo 48354 / 2007 / Projeto / Título: Otimização Dinâmica de uma Planta de Gaseificação para Geração de Gás Combustível./ Edital CNPq 15 / 2007 - Universal). 


\section{Referências}

[1] Sundac, Nikola., Catalytic Cracking of tar from biomass gasification. Department of Chemical Engineering,, Lund University, P. O. Box 124, SE-221 00 Lund, Sweden

[2] Devi, L., Ptasinski, K.J., Janssen, F.J.J.G, A review of primary measures for tar elimination in biomass gasification process, Biomass and Bioenergy, Vol. 24., pp. 125-140 (2003).

[3] Lamacz, A.; Krzton, A.; Musi, A.; Da Costa, P.; Reforming of Model Gasification Tar Compounds. Catalysis Letters,V. 128, Numbers 1-2, pp. $40-48$.

[4] Swierczynski, D.; Courson, C.; Kiennemann, A Study of steam reforming of toluene used as model compound of tar produced by biomass gasification. Chemical Engineering and Processing, v. 47, p. 508-513, 2007.

[5] Milne T.A., Evans R.J., Biomass Gasifier "Tars": Their Nature, Formation and Conversion, NREL/TP-570-25357(1998).

[6] Castro, A.J.A., Cassiano, D.A., Ferreira, N.L., Gaseificação do bagaço de cana- eaçucar: modelagem, análise e comparação com sistema real. Exacta, v. 7, pp 39-47 (2009).

\section{Apendice A}

Tabela A1: Parâmetros $\alpha$ para o sistema de equações

\begin{tabular}{|l|l|}
\hline$\alpha_{1, \mathrm{v}}=\frac{\mathrm{V}_{\mathrm{v}}}{\left(1-\varepsilon_{\mathrm{s}}\right)}$ & $\alpha_{12, \mathrm{v}}=\frac{2 \Delta \mathrm{z} \alpha_{8, \mathrm{v}}}{2+\Delta \mathrm{z} \alpha_{8, \mathrm{v}}}$ \\
\hline$\alpha_{2, \mathrm{v}}=\frac{\lambda_{\mathrm{ax}, \mathrm{v}}}{\rho_{\mathrm{v}} \mathrm{C}_{\mathrm{p}, \mathrm{v}}}$ & $\begin{array}{l}\alpha_{13, \mathrm{v}}=\alpha_{5, \mathrm{v}} \alpha_{9, \mathrm{v}}+\alpha_{6, \mathrm{v}} \alpha_{11, \mathrm{v}} \\
+\alpha_{7, \mathrm{v}}\end{array}$ \\
\hline$\alpha_{3, \mathrm{v}}=\frac{\alpha_{\mathrm{vs}} \eta_{\mathrm{CE}} \varepsilon_{\mathrm{s}} \mathrm{a}_{\mathrm{s}}}{\left(1-\varepsilon_{\mathrm{s}}\right) \rho_{\mathrm{v}} \mathrm{C}_{\mathrm{p}, \mathrm{v}}}$ & $\begin{array}{l}\alpha_{14, \mathrm{v}}=\alpha_{6, \mathrm{v}} \alpha_{12, \mathrm{v}} \\
\alpha_{5, \mathrm{v}} \alpha_{10, \mathrm{v}}\end{array}$ \\
\hline$\alpha_{4, \mathrm{v}}=\frac{\alpha_{\mathrm{vw}}\left(1-\eta_{\mathrm{EC}}\right) \mathrm{a}_{\mathrm{w}}}{\left(1-\varepsilon_{\mathrm{s}}\right) \rho_{\mathrm{v}} \mathrm{C}_{\mathrm{p}, \mathrm{v}}}$ & $\alpha_{15, \mathrm{~s}}=\frac{\lambda_{\mathrm{ax}, \mathrm{s}}}{\rho_{\mathrm{s}} \mathrm{C}_{\mathrm{p}, \mathrm{s}}}$ \\
\hline
\end{tabular}

\begin{tabular}{|c|c|}
\hline$\alpha_{5, v}=\frac{\alpha_{2, v}}{(\Delta z)^{2}}-\frac{\alpha_{1, v}}{2 \Delta z}$ & 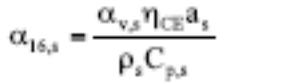 \\
\hline$\alpha_{6, v}=\frac{\alpha_{2, v}}{(\Delta z)^{2}}+\frac{\alpha_{1, v}}{2 \Delta z}$ & $\alpha_{17, s}=\frac{r_{x}^{\prime}\left(-\Delta H_{t}\right)}{\rho_{s} C_{p, s}}$ \\
\hline $\begin{array}{l}\alpha_{7, v}=\alpha_{4, \gamma}-\alpha_{3, v}- \\
\frac{2 \alpha_{2, v}}{(\Delta z)^{2}}\end{array}$ & $\alpha_{1 S, s}=\frac{\alpha_{1 S s}}{(\Delta Z)^{2}}$ \\
\hline$\alpha_{s, v}=\frac{\rho_{v} C_{p, v} V_{v}}{\left(1-\varepsilon_{s}\right) \lambda_{e x, v}}$ & $\alpha_{19, s}=\left[\frac{-2 \alpha_{15, s}}{(\Delta Z)^{2}}-\alpha_{16, s}\right.$ \\
\hline$\alpha_{9, x}=\frac{2+\Delta z \alpha_{s, r}}{2-\Delta z \alpha_{s, y}}$ & $\alpha_{20, s}=\frac{-q_{e} \Delta Z}{\varepsilon_{s} \lambda_{\text {ars }}}$ \\
\hline$\alpha_{10, v}=\frac{2 \Delta z \alpha_{g, v}}{2-\Delta z a_{g, v}}$ & $\alpha_{21, s}=2 \alpha_{18, s}+\alpha_{19, s}$ \\
\hline$\alpha_{11, v}=\frac{2-\Delta z \alpha_{g, v}}{2+\Delta z \alpha_{g, r}}$ & 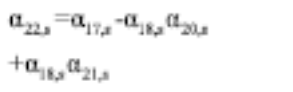 \\
\hline
\end{tabular}

Tabela A2: Parâmetros $\alpha_{\mathrm{t}}$ para o sistema de equações

\begin{tabular}{|c|c|c|}
\hline$\alpha_{L, 1}=\frac{D_{1}}{h_{1}}$ & \multicolumn{2}{|c|}{$a_{7, t}=\frac{4 Q_{8}}{\pi d^{2} D_{1}}$} \\
\hline$a_{2,4}=\frac{\varepsilon_{z} M_{t} R_{t}}{\mathbf{h}_{t}}$ & $a_{3, t}=$ & $\frac{2+\boldsymbol{\alpha}_{7, t} \Delta z}{2-\alpha_{T, t} \Delta z}$ \\
\hline$\alpha_{3,1}=\frac{4 Q_{\mathrm{L}}}{\mathbf{h}_{\mathrm{t}} \pi \mathrm{d}^{2}}$ & $a_{p, t}=$ & $\left.\frac{2 u_{7, t} \Delta z}{2-\alpha_{7, t} \Delta z}\right]$ \\
\hline$\alpha_{u_{1}, 1}=\left[\frac{\alpha_{L, t}}{\Delta z^{2}}-\frac{\alpha_{1,1}}{2 \Delta z}\right]$ & $\alpha_{10, \mathrm{t}}=$ & $\frac{2-a_{T, t} \Delta z}{2+a_{P, 1} \Delta z}$ \\
\hline$\alpha_{S, t}=\left[\frac{\alpha_{1,4}}{\Delta z^{2}}+\frac{\alpha_{1,1}}{2 \Delta z}\right]$ & $\alpha_{11, \mathrm{t}}=$ & $\frac{2 a_{1, \lambda z} \Delta z}{2+a_{7,1} \Delta z}$ \\
\hline$u_{6, t}=\frac{2 u_{1,1}}{\Delta z^{2}}$ & $\begin{array}{l}\alpha_{12,1}= \\
\alpha_{5,} \alpha_{1}\end{array}$ & $\begin{array}{l}\alpha_{4,1} \alpha_{8, t}+ \\
\alpha_{0,1}-\alpha_{0, t}\end{array}$ \\
\hline$\alpha_{13,1}=$ & $a_{2 t}$ & $2_{4,1} \alpha_{9, t}$ \\
\hline
\end{tabular}


Tabela A3: Parâmetros $\alpha_{\mathrm{O}_{2}}$ para o sistema de

\begin{tabular}{|c|c|}
\hline$a_{1, \rho_{2}}=\frac{D_{0_{2}}}{h_{Q_{2}}}$ & $a_{T, Q_{1}}=\frac{4 Q_{\mathrm{g}}}{\pi d^{2} \mathrm{D}_{0_{2}}}$ \\
\hline$\alpha_{2, O_{3}}=\frac{{ }_{s_{5}} \mathrm{M}_{\mathrm{O}_{2}} \mathbf{R}_{\mathrm{O}_{3}}}{\mathbf{h}_{\mathrm{O}_{2}}}$ & $e_{8, O_{3}}=\left[\frac{2+\alpha_{10_{2} \Delta z}}{2-\alpha_{30_{2} \Delta z} \Delta z}\right.$ \\
\hline$a_{3 \mathrm{O}_{1}}=\frac{4 \mathrm{Q}_{\mathrm{E}}}{\mathrm{h}_{\mathrm{O}_{2}} \pi \mathrm{d}^{2}}$ & $a_{9, O_{1}}=\left[\frac{2 a_{1,0_{1}} \Delta z}{2-a_{1,0_{2}} \Delta z}\right.$ \\
\hline$a_{4 \rho_{1}}=\left[\frac{a_{1, \rho_{2}}}{\Delta z^{2}}-\frac{a_{3, \rho_{2}}}{2 \Delta z}\right.$ & $a_{10, Q_{2}}=\left[\frac{2-\alpha_{7,0_{2}} \Delta z}{2+a_{7,0_{2}} \Delta z}\right]$ \\
\hline$a_{s, O_{1}}=\left[\frac{\alpha_{1, \rho_{2}}}{\Delta z^{2}}+\frac{\alpha_{3, \rho_{2}}}{2 \Delta z}\right.$ & $a_{11, Q_{2}}=\left[\frac{2 \alpha_{7, O_{2}} \Delta z}{2+\alpha_{7,0_{2}} \Delta z}\right]$ \\
\hline$\alpha_{6,0_{2}}=\frac{2 \alpha_{1, \rho_{1}}}{\Delta z^{2}}$ & $\begin{array}{l}\alpha_{12, O_{2}}=\alpha_{4, O_{2}} \alpha_{8, O_{2}}+ \\
a_{5, O_{2}} a_{10,0_{2}}-a_{6, O_{2}}\end{array}$ \\
\hline
\end{tabular}

Tabela A5: Parâmetros $\alpha_{\mathrm{co}}$ para o sistema de equações

\begin{tabular}{|c|c|c|}
\hline$a_{1, \infty 0}=\frac{\mathrm{D}_{\infty 0}}{\mathbf{h}_{\infty}}$ & \multicolumn{2}{|c|}{$\alpha_{7,00}=\frac{4 Q_{g}}{\pi d^{2} D_{C 0}}$} \\
\hline$a_{200}=\frac{\varepsilon_{x} \mathbf{M}_{\infty 0} \mathbf{R}_{\infty 0}}{\mathbf{h}_{\infty 0}}$ & $\alpha_{8,00}=$ & {$\left[\frac{2+\alpha_{T, \infty} \Delta z}{2-\alpha_{7, \infty 0} \Delta z}\right.$} \\
\hline$a_{3, C O}=\frac{4 Q_{g}}{h_{\infty} \pi d^{2}}$ & $\alpha_{2,00}=$ & $\frac{2 \alpha_{1, \infty} \Delta z}{2-\alpha_{7, \infty} \Delta z}$ \\
\hline$a_{4,00}=\left[\frac{\alpha_{1,00}}{\Delta z^{2}}-\frac{a_{3,00}}{2 \Delta z}\right.$ & $\alpha_{10, C O}=$ & {$\left[\frac{2-a_{2,00} \Delta z}{2+a_{7,00} \Delta z}\right]$} \\
\hline$a_{5, \infty 0}=\left[\frac{u_{1, \infty 0}}{\Delta z^{2}}+\frac{u_{3, \infty 0}}{2 \Delta z}\right.$ & $\alpha_{11, C O}=$ & {$\left[\frac{2 u_{1,00} \Delta z}{2+a_{1,00} \Delta z}\right]$} \\
\hline$\alpha_{0,00}=\frac{2 \alpha_{1,00}}{\Delta z^{2}}$ & \multicolumn{2}{|c|}{$\begin{array}{l}\alpha_{12, \infty 0}=\alpha_{4, c 0} \alpha_{8, \infty 0}+ \\
\alpha_{5, c 0} \alpha_{10, c 0}-\alpha_{6, \infty 00}\end{array}$} \\
\hline$a_{13,00}=$ & $a_{2, \infty 0}$ & $\alpha_{4 \infty} \alpha_{9,00}$ \\
\hline
\end{tabular}

Tabela A4: Parâmetros $\alpha_{\mathrm{H}_{2}}$ para o sistema de equações

\begin{tabular}{|c|c|c|}
\hline$a_{1, \mathrm{H}_{2}}=\frac{\mathrm{D}_{\mathrm{B}_{2}}}{\mathrm{~h}_{\mathrm{B}_{2}}}$ & \multicolumn{2}{|c|}{$\alpha_{\tau, H_{2}}=\frac{4 Q_{E}}{\pi d^{2} D_{H_{2}}}$} \\
\hline$\alpha_{2, \mathrm{~B}_{2}}=\frac{\varepsilon_{s} \mathrm{M}_{\mathrm{H}_{2}} \mathrm{R}_{\mathrm{H}_{2}}}{\mathrm{~h}_{\mathrm{H}_{2}}}$ & $a_{3, \mathrm{H}_{2}}=$ & $\frac{2+\alpha_{7, \mathrm{H}_{2}} \Delta \mathrm{z}}{2-\alpha_{9 \mathrm{H}_{2}} \Delta \mathrm{z}}$ \\
\hline$\alpha_{3, H_{2}}=\frac{4 Q_{z}}{h_{B_{2}} \pi d^{2}}$ & $\alpha_{9 H_{2}}=$ & $\left.\frac{2 \alpha_{T, \mathrm{H}_{y}} \Delta z}{2-\alpha_{3, \mathrm{H}_{2}} \Delta z}\right]$ \\
\hline$\alpha_{4, \mathrm{~B}_{2}}=\left[\frac{\alpha_{1, \mathrm{~B}_{3}}}{\Delta \mathrm{z}^{2}}-\frac{\alpha_{1, \mathrm{H}_{2}}}{2 \Delta z}\right]$ & $\alpha_{10, H_{1}}=$ & {$\left[\frac{2-\alpha_{7, H_{2}} \Delta z}{2+\alpha_{7, H_{2}} \Delta z}\right]$} \\
\hline$\alpha_{S, \mathrm{~B}_{2}}=\left[\frac{\alpha_{1, \mathrm{~B}_{2}}}{\Delta z^{2}}+\frac{\alpha_{2, \mathrm{H}_{2}}}{2 \Delta z}\right]$ & $\alpha_{11, \mathrm{~B}_{2}}=$ & {$\left[\frac{2 e_{7, B_{2}} \Delta z}{2+\alpha_{7, B_{2}} \Delta z}\right]$} \\
\hline$\alpha_{6, H_{2}}=\frac{2 a_{1, H_{2}}}{\Delta z^{2}}$ & $\begin{array}{l}\alpha_{12, \mathrm{~B}_{2}}= \\
\alpha_{3, \mathrm{~B}_{2}} \alpha_{1}\end{array}$ & $\begin{array}{l}=\alpha_{4, \mathrm{~B}_{2}} \alpha_{3, \mathrm{~B}_{2}}+ \\
\mathrm{H}_{1}-\alpha_{6, \mathrm{~B}_{2}}\end{array}$ \\
\hline$\alpha_{13, \mathrm{~B}_{2}}=\alpha_{5, \mathrm{~B}_{2}} \alpha_{11,}$ & $\alpha_{2, \mathrm{~B}_{2}}$ & $-\alpha_{4, H_{2}} \alpha_{9, \mathrm{~B}_{2}}$ \\
\hline
\end{tabular}

